\title{
A Merged Molecular Representation Learning for Molecular Properties Prediction with a Web-based Service
}

Hyunseob Kim

Korea Institute of Science \& Technology Information

Jeongcheol Lee

Korea Institute of Science \& Technology Information

Sunil Ahn

Korea Institute of Science \& Technology Information

Jongsuk Lee ( $\nabla$ jsruthlee@kisti.re.kr)

Korea Institute of Science \& Technology Information

\section{Research Article}

Keywords: dramatic development, molecular property, drug discovery, fingerprint, SMILES, graphs

Posted Date: December 21st, 2020

DOI: https://doi.org/10.21203/rs.3.rs-130308/v1

License: (9) This work is licensed under a Creative Commons Attribution 4.0 International License.

Read Full License 


\title{
A Merged Molecular Representation Learning for Molecular Properties Prediction with a Web-based Service
}

\author{
Hyunseob Kim ${ }^{1}$, Jeongcheol Lee ${ }^{1}$, Sunil Ahn ${ }^{1}$, and Jongsuk Ruth Lee ${ }^{1, *}$ \\ ${ }^{1}$ Center for Computational Science Platform, Korea Institute of Science and Technology Information, Daejeon, \\ 34141, Republic of Korea \\ *jsruthlee@kisti.re.kr
}

\begin{abstract}
Deep learning has brought a dramatic development in molecular property prediction which is crucial in the field of drug discovery. Various methods such as fingerprint, SMILES, graphs have been proposed for representing molecules. Recently, unlabeled molecule data is used to improve performance for various pre-training methods. The main challenge of molecular properties predictions is designing a data representation and model that can show good performance for various datasets. However, performance deviation due to scarcity of dataset exists in constructing the model. We propose a new self-supervised method to learn the characteristics and structures of molecules by integrating existing methods. The key of our model is learning structures with matrix embedding and learning logics that can infer descriptors via QED prediction. As a result, our method improves the generalization of the data and achieves the best average performance by benchmarking downstream tasks. Moreover, we develop a web-based fine-tuning service to utilize our model on various tasks.
\end{abstract}

\section{Introduction}

Drug discovery has traditionally been a very expensive research field. There are lots of candidates to validate and the process of validation is significantly complex and long. Recently, deep learning ${ }^{1-3}$ is utilized to increase efficiency and accelerate the overall process in drug discovery such as molecular property prediction, virtual screening, etc. However, there are still some obstacles to overcome in order to apply it to the actual industry. Despite the presence of tremendous molecules, labeled datasets are scarce and classified with detailed purposes. Those characteristics of the dataset cause limitations such as overfitting and unstable model performance in evaluating models.

Self-supervised learning ${ }^{4-6}$ which do not need labeled data for training shows performance improvements in most tasks of natural language processing (NLP) by pre-training the model such as Transformer ${ }^{7}$. Those methods build a huge language model that can be utilized to various tasks using the pre-training. It demonstrates a generalized model can be constructed with large-scale data and fine-tuning works on downstream tasks. This attempt also appears to solve molecular property predictions in drug discovery. Methods focused on learning molecular structures with a large amount of data have been proposed in Transformer and graph neural networks ${ }^{8}$ (GNN). They show well-designed pre-training strategies reflecting general characteristics of molecule data to improve overall performance on downstream tasks.

Molecules can be converted to various kinds of data representations. Traditionally, fingerprint ${ }^{9,10}$ and descriptors are used as input features in constructing models. Simplified Molecular-Input Line-Entry System (SMILES) ${ }^{11,12}$ and graph $^{13,14}$ representations are usually used with neural networks and show improvements by constructing novel architectures. Convolutional Neural Networks (CNN) and Graph Convolutional Networks (GCN) work effectively on molecular properties prediction. Interestingly, we can find that each dataset has a suitable data representation showing better performance from previous works. In other words, some kinds of representation are highly dependent on a specific task and cannot extract general knowledge from data. Therefore, it is necessary to find the data representation which does not have a high dependency on tasks and shows stable performance about various prediction tasks.

The molecular fingerprint is a popular method representing substructures. It encodes the structure of a molecule by using a hash function. Machine learning models use them as features with additional descriptors such as Partition coefficient (logP), Hydrogen Bond Acceptors (HBA), Hydrogen Bond Donors (HBD), Polar Surface Area (PSA), etc. However, fingerprints reveal limitations caused by the hash function in predicting properties. For solving those limitations, a neural networks-based fingerprint appears. Seq2seq fingerprint ${ }^{15}$ learns molecular embedding with recurrent neural networks (RNN). SMILES is a text representation of molecules in a single line. As it is sequential and composed of text, methods inspired by NLP such as word embedding ${ }^{16}, \mathrm{RNN}^{17,18}$ have been proposed. Mol2 $\mathrm{Vec}^{19}$ is a molecular representation inspired by word2vec. It overcomes the 
drawbacks of fingerprint such as bit collisions. Smiles2 Vec ${ }^{18}$ learns a representation of a molecule using RNN on SMILES tokens.

Recently, the Transformer which records state-of-the-art in NLP has been applied to molecular property prediction. SMILESTransformer ${ }^{20}$ extracts latent vectors from the output of teh encoder and uses it as a fingerprint of a molecule. It is superior to others in small-data settings. SMILES-BERT ${ }^{21}$ applies a pre-training task of BERT ${ }^{5}$ on SMILES and shows improved performance in several prediction tasks. Molecule Attention Transformer ${ }^{22}$ (MAT) unifies atomic features with structural information and augments attention weights with graph structure and distance. Those works demonstrate pre-training on the Transformer is valid on molecular data.

GNN records state-of-the-art on various prediction tasks. In addition to existing models, new models ${ }^{23-25}$ and pre-training methods considering chemical properties are also emerging. GCN is successfully applied to molecular data with improving performance. Multi-channel approaches ${ }^{26-28}$ show better performance than single graph-based models. They learn the substructures of molecules by constructing a model for each feature such as an atom, bond, and edge. Pre-training strategies ${ }^{8}$ is also designed to find substructures and properties. Context prediction and supervised attribute prediction are designed to train the model in both node-level and graph-level. They help to predict substructures and neighborhood of nodes on molecule data.

In this paper, we propose a new self-supervised method for pre-training. Our method is based on masking \& recovery pre-training task that predicts masked tokens of SMILES using a Transformer. Additionally, we design the matrix embedding layer which can learn structures of molecules and add a Quantitative Estimation of Drug-likeness (QED) value prediction task in the pre-training step. The matrix embedding layer complements the connectivity of molecules which can be lost in SMILES. The QED prediction task helps to infer descriptors that are composing QED and solving downstream tasks. As a result, our model learns a merged data representation for molecules in the pre-training step.

We perform benchmarking on 10 molecule datasets from MoleculeNet ${ }^{29}$. Each downstream task is performed by fine-tuning the pre-trained model. Our pre-training method improves the generalization of molecules compared with various representations. We demonstrate our model achieves the best average performance on both classification and regression tasks. Finally, we develop a fine-tuning service on the web-based server. It can learn various molecular properties prediction tasks using a pre-trained model. Users can easily get stable and high performance results without constructing task-specific architectures.

\section{Methods}

\section{A merged molecular representation}

Our self-supervised method for learning merged molecular representation is based on pre-training tasks and the architecture of BERT. For integrating various representations, we propose a matrix embedding layer and QED prediction task. The overall architecture is shown in Figure 1. The adjacency matrix of input SMILES goes to the matrix embedding layer to produce a matrix embedding vector. After that, input SMILES is split to tokens and converted to a token embedding vector in the embedding layer. Input tokens are fed to the Transformer including masked tokens. The outputs of the Transformer consist of a QED value and recovered input tokens. Finally, the model performs QED prediction and prediction on masked tokens.

The Transformer is an encoder-decoder structure which can process the sequential input data. We use only Transformerencoder in constructing the model. The encoder mainly consists of self-attention block and feed-forward networks. The self-attention means a mechanism relating different positions of a single sequence in order to compute a representation of the same sequence. The self-attention block has Multi-Head Attention layer and computes attention weights as followed:

$$
\begin{aligned}
& A(Q, K, V)=\operatorname{softmax}\left(\frac{Q K^{T}}{\sqrt{d_{k}}}\right) V \\
& Q=H W^{Q}, K=H W^{K}, V=H W^{V}
\end{aligned}
$$

where $Q$ is the query matrix, $K$ is the key matrix, $V$ is the value matrix and $d_{k}$ is the dimension of queries and keys. $Q, K$, and $V$ are the same sequential input for computing the self-attention. $Q, K, V$ are a multiplication of input hidden state $H$ and the weight matrix $W^{Q}, W^{K}, W^{V}$. The softmax function is applied to obtain the attention weights. It is called Scaled Dot-Product Attention. Multi-Head Attention computes the value of several Scaled Dot-Product Attention layers in parallel. Fully connected feed-forward network is connected with the output of the self-attention block. This process is repeated as the number of layers.

The masked language model which is used in BERT predicts masked words for the given sentence. It is a self-supervised method in NLP using unlabeled data. We apply it to predict masked tokens of given SMILES from unlabeled molecular data. For masking, we tokenize the SMILES string and randomly choose $15 \%$ on all tokens of input data. We replace a chosen token to $<$ MASK $>$ with $80 \%$. For the rest, it is replaced with another token in the dictionary with $10 \%$ or unchanged with $10 \%$. This process is designed to prevent overfitting in pre-training. 


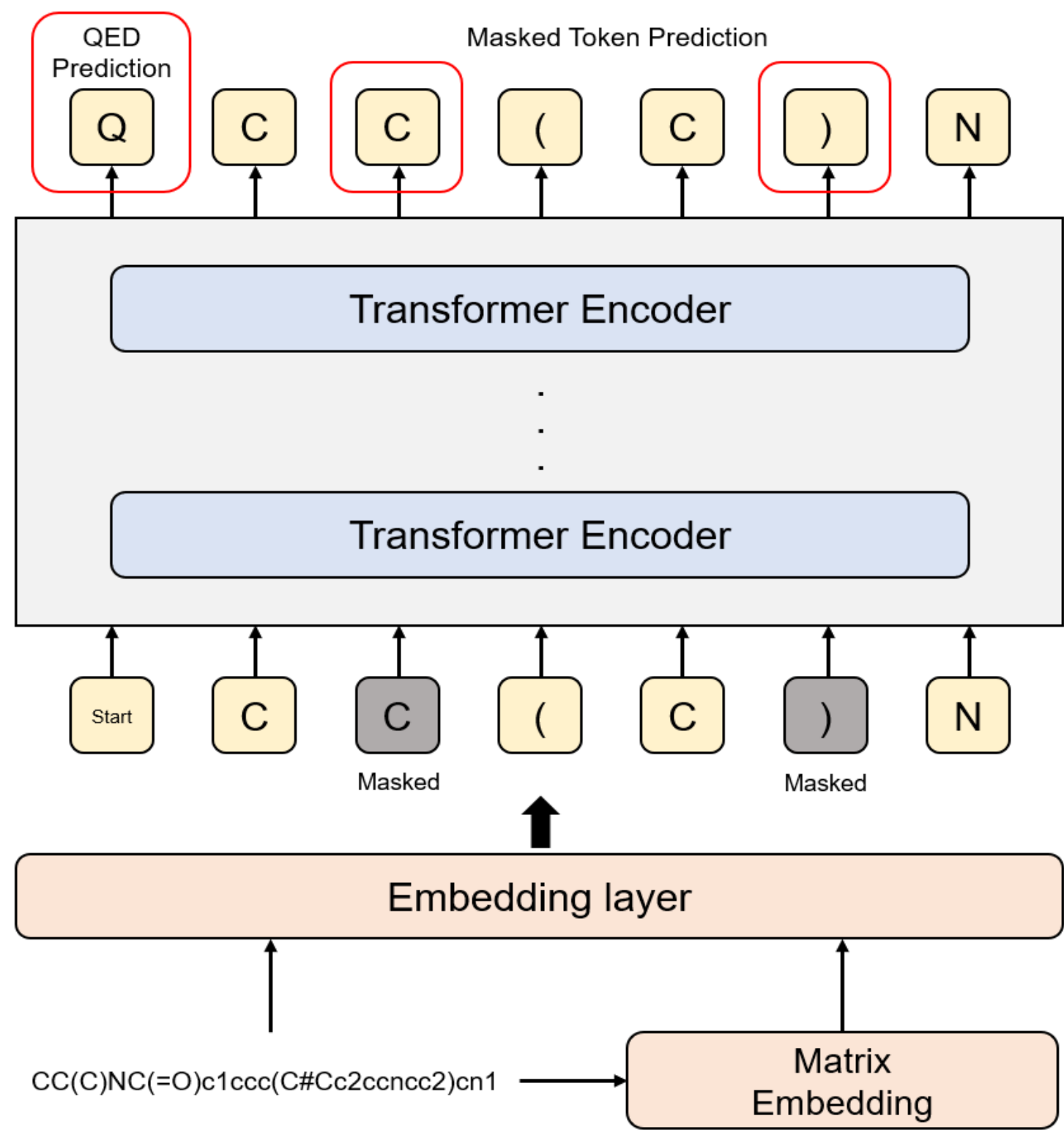

Figure 1. The overview of our pre-training methods and model. The input SMILES is converted to input vectors via embedding layer. The red boxes are targets of pre-training among the outputs of Transformer. The fine-tuning uses the same architectures except output layers.

However, SMILES is insufficient to represent the connectivity and substructures of molecules. Therefore, we design the matrix embedding layer to complement structural information as followed:

$$
E(A)=W_{e} A W_{a}+b
$$

where $W_{a}$ is the weight matrix for an adjacency matrix $A, W_{e}$ is the weight vector for generating an embedding token and $b$ is the bias vector. The matrix embedding layer learns the adjacency matrix of molecules and produces a vector for embedding. The adjacency matrix is useful information in prediction, but adding a matrix embedding vector on unrelated tokens disturbs training. Therefore, the matrix embedding vector produced from this layer is only applied to atomic tokens which are related to an adjacency matrix. As a result, the input SMILES is converted to the sum of token embedding, position embedding, and matrix embedding shown as Figure 2. Position embedding notifies the sequence of input data. The start and end token are included in the input data to distinguish the length of SMILES more clearly.

BERT uses two sentences as input data and performs the next sentence prediction task. It helps BERT to understand the 


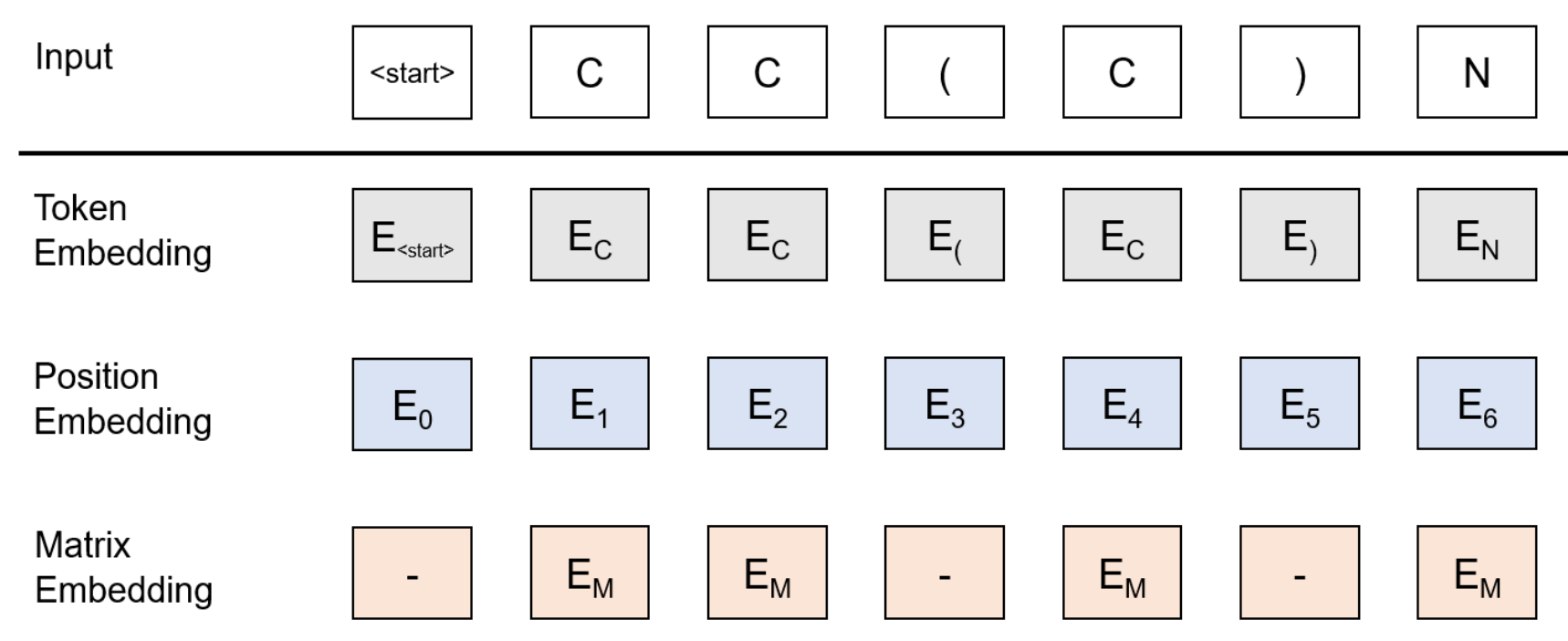

Figure 2. The input representation of SMILES. The input is the sum of the token embeddings, the position embeddings, and the matrix embeddings. The matrix embedding is only applied to atomic tokens.

context of documents. Similarly, our model performs QED $^{30}$ prediction as a second pre-training task to learn the properties of molecules. QED indicates the drug-likeness of molecules and reflects molecular properties such as logP, molecular weight, the number of aromatic rings, topological polar surface area, rotatable bonds, etc. It also reflects the underlying distribution of molecular properties and is calculated as a value between 0 to 1 considering the properties of the molecule as followed:

$$
Q E D=\exp \left(\frac{1}{n} \sum_{i=1}^{n} \ln d_{i}\right)
$$

where $d_{i}$ is a desirability function of each property. We expect the model constructs a logic that can infer properties and descriptors of the molecule during pre-training. By predicting QED value, the model can predict descriptors and merge those properties in the output representation. We apply a linear classifier on the first output of the Transformer for prediction. In fine-tuning, this mechanism helps to predict descriptors and solving downstream tasks.

Additionally, we develop the service to utilize our pre-trained model on new datasets. It can accelerate the model development process with a user-friendly web-based interface. The service is operated with the following components.

- Data submission : Users can submit a prepared dataset and select the task type.

- Fine-tuning : Begin the fine-tuning with the pre-trained model. The result of training will be delivered to the viewer.

- Result viewer : The viewer shows the result of training including performances, graphs and details of the model.

We will address contributions of the service in Results and Discussion.

\section{Experimental settings}

In pre-training, we use 9 million unlabeled molecules selected from $\mathrm{ZINC}^{31}$ database. We test on the effect of pre-training data size from 2 million to 9 million. We see that the validation performance gets higher as data size increases. The model which used 9 million data records $94.4 \%$ accuracy on masked tokens prediction and 0.02 Mean Absolute Error (MAE) on QED prediction. Therefore, we decide to use 9 million data for pre-training. We use Transformer Encoder with 8 layers and 16 attention heads. The activation function used in the Transformer is Gaussian Error Linear Units (GELU) ${ }^{32}$. The maximum input sequence length is 256 . The input embedding vector and hidden units of the Transformer are 1024 dimensions. We do not apply dropout on the entire model which causes underfitting in this work. We use Adam ${ }^{33}$ optimizer with the scheduled learning rate as followed:

$$
\text { learning_rate }=0.1 \cdot d_{m o d e l}^{-0.5} \cdot \min \left(s t e p \_n u m^{-0.5}, \text { step_num } \cdot \text { warmup_steps }^{-1.5}\right)
$$

We use 10000 as warm-up steps. The loss function for pre-training is the sum of Mean Squared Error and Cross-entropy. Mean Squared Error is used to train the QED value prediction and Cross-entropy is used to train the masked token prediction. 


\begin{tabular}{cccc}
\hline Dataset & Tasks & \# Molecules & Metric \\
\hline \hline BBBP & 1 & 2039 & ROC-AUC \\
Tox21 & 12 & 7831 & ROC-AUC \\
ToxCast & 617 & 8575 & ROC-AUC \\
SIDER & 27 & 1427 & ROC-AUC \\
ClinTox & 2 & 1478 & ROC-AUC \\
MUV & 17 & 93087 & ROC-AUC \\
HIV & 1 & 41127 & ROC-AUC \\
BACE & 1 & 1513 & ROC-AUC \\
Esol & 1 & 1128 & RMSE \\
Freesolv & 1 & 642 & RMSE
\end{tabular}

Table 1. Information of dataset from MoleculeNet.

As downstream tasks, we select 10 datasets from MoleculeNet ${ }^{29}$ including classification and regression shown as Table 1. Classification tasks include BBBP ${ }^{34}$, Tox $21^{35}$, ToxCast $^{36}, \operatorname{SIDER}^{37}, \mathrm{ClinTox}{ }^{38}, \mathrm{MUV}^{39}, \mathrm{HIV}, \mathrm{BACE}^{40}$ and regression tasks include $\mathrm{ESOI}^{41}, \mathrm{Freesolv} \mathrm{v}^{42}$. Datasets which have multiple tasks are learned jointly in a single model.

- BBBP: Blood-brain barrier penetration. Prediction of the barrier permeability.

- Tox21: Qualitative toxicity measurements on 12 different targets, including nuclear receptors and stress response pathways.

- ToxCast: Data collection providing toxicology data based on in vitro high-throughput screening.

- SIDER: Drug side-effects into 27 system organ classes following MedDRA classification.

- ClinTox: It contains clinical trial toxicity and FDA approval status.

- MUV: Benchmark dataset from PubChem BioAssay. It contains 17 challenging tasks for validation of virtual screening techniques.

- HIV: The ability to inhibit HIV replication.

- BACE: Binding results for a set of inhibitors of human $\beta$-secretase 1.

- Esol: Water solubility data.

- Freesolv: Hydration free energy of small molecules in water.

In fine-tuning, we apply scaffold splitting into classification tasks and random splitting on regression tasks. Scaffold splitting is effective in performing out-of-distribution samples. Molecules are processed with RDKit library. Our model has trained with Adam optimizer with a learning rate of $\{0.00001,0.00005\}$, and 15 epochs. We use a batch size of $\{64,128\}$. The split ratio of train/valid/test is $80 \%: 10 \%: 10 \%$. We evaluate classification tasks using ROC-AUC and regression tasks using Root Mean Square Error (RMSE). We take the average ROC-AUC on datasets that have multiple tasks. An early stopping process based on the best validation score is applied to all tasks. We record the average performance of 5 times.

\section{Results and Discussion}

We select four methods as baselines to compare the various representation of molecules. RandomForest ${ }^{43}$ is a traditional approach using Extended Connectivity Fingerprint (ECFP) ${ }^{10}$ and descriptors of molecule. Graph Isomorphism Networks $(\mathrm{GIN})^{44}$ is non-pretrained graph-based model. GIN is a kind of message-passing neural networks that is implemented to conduct Weisfeiler-Lehman (WL) graph isomorphism test. GIN shows better performance on distinguishing topological identical than other GNNs such as GCN. As a pre-trained model, Pretrained-GIN and SMILES-BERT are used to compare. Pretrained-GIN is pre-trained by context prediction and graph-level supervised learning. SMILES-BERT follows the concept of BERT and is pre-trained by masked token prediction. By including pre-trained models, we compare and evaluate our method against diverse pre-training strategies.

We compare the performance of our model with various data representations. We report results on molecular properties predictions in Table 2, 3. In case of RandomForest, it shows competitive results on classification tasks. We can infer descriptors 


\begin{tabular}{c|ccccccccc}
\hline & BBBP & Tox21 & ToxCast & SIDER & ClinTox & MUV & HIV & BACE & Avg \\
\hline \hline RandomForest & $\mathbf{7 4 . 7} \pm \mathbf{0 . 5}$ & $76.8 \pm 0.1$ & $65.3 \pm 0.2$ & $\mathbf{6 4 . 8} \pm \mathbf{0 . 6}$ & $78.5 \pm 1.3$ & $65.9 \pm 2.0$ & $77.8 \pm 1.1$ & $83.0 \pm 0.6$ & 73.4 \\
GIN & $65.8 \pm 4.5$ & $74.0 \pm 0.8$ & $63.4 \pm 0.6$ & $57.3 \pm 1.6$ & $58.0 \pm 4.4$ & $71.8 \pm 2.5$ & $75.3 \pm 1.9$ & $70.1 \pm 5.4$ & 67.0 \\
Pretrained-GIN & $68.7 \pm 1.3$ & $\mathbf{7 8 . 1} \pm \mathbf{0 . 6}$ & $\mathbf{6 5 . 7} \pm \mathbf{0 . 6}$ & $62.7 \pm 0.8$ & $72.6 \pm 1.5$ & $\mathbf{8 1 . 3} \pm \mathbf{2 . 1}$ & $\mathbf{7 9 . 9} \pm \mathbf{0 . 7}$ & $\mathbf{8 4 . 5} \pm \mathbf{0 . 7}$ & 74.2 \\
SMILES-BERT & $73.3 \pm 1.0$ & $75.8 \pm 0.6$ & $61.5 \pm 0.9$ & $61.8 \pm 0.3$ & $97.9 \pm 0.8$ & $71.1 \pm 1.6$ & $76.6 \pm 1.1$ & $80.0 \pm 3.3$ & 74.8 \\
Our model & $72.4 \pm 0.9$ & $77.4 \pm 0.5$ & $\mathbf{6 5 . 3} \pm \mathbf{1 . 1}$ & $63.1 \pm 0.6$ & $\mathbf{9 9 . 0} \pm \mathbf{0 . 3}$ & $73.2 \pm 1.7$ & $77.6 \pm 1.6$ & $82.0 \pm 1.7$ & $\mathbf{7 6 . 2}$
\end{tabular}

Table 2. ROC-AUC (\%) performance on classification tasks. The best score of each dataset is marked as bold.

\begin{tabular}{c|cc|c}
\hline & Esol & Freesolv & Avg \\
\hline \hline RandomForest & $1.279 \pm 0.127$ & $2.419 \pm 0.293$ & 1.849 \\
GIN & $0.731 \pm 0.092$ & $1.171 \pm 0.197$ & 0.951 \\
Pretrained-GIN & $0.605 \pm 0.064$ & $1.112 \pm 0.127$ & 0.858 \\
SMILES-BERT & $0.473 \pm 0.052$ & $0.810 \pm 0.088$ & 0.641 \\
Our model & $\mathbf{0 . 4 5 1} \pm \mathbf{0 . 0 3 3}$ & $\mathbf{0 . 7 5 5} \pm \mathbf{0 . 0 7 8}$ & $\mathbf{0 . 6 0 3}$
\end{tabular}

Table 3. RMSE on regression tasks. The best score of each dataset is marked as bold.

are still effective in learning classification tasks and our QED prediction task is designed to reflect it. Our model shows the best average performance in both classification and regression. We see that the average ROC-AUC of our model achieves $1.4 \%$ over SMILES-BERT and 2\% over Pretrained-GIN on classification tasks shown as Table 2. In particular, our model shows improvements and stable performance with the lowest variance on regression tasks.

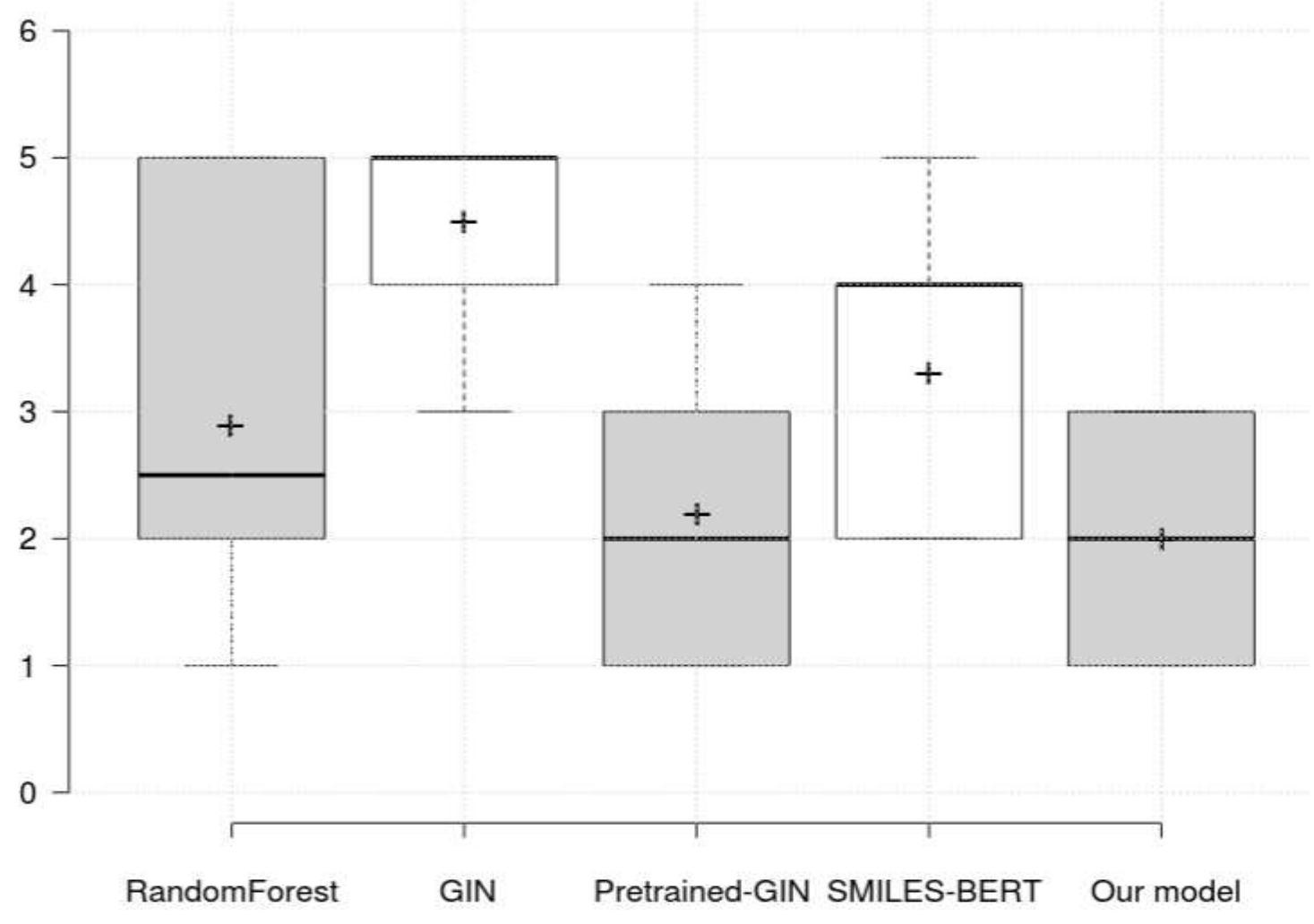

Figure 3. The average rank across the 10 datasets from MoleculeNet. Our model outperforms other models. Interestingly, RandomForest records higher average rank than GIN and SMILES-BERT.

We report the average rank of models shown as Figure 3. Our model shows good performance evenly on downstream tasks. However, other models have downstream tasks that record significantly low performance. RandomForest records low performance on regression tasks and MUV. Graph representation models especially show low performance on BBBP, 
ClinTox, despite outperforming on other tasks. On the contrary, our model does not have tasks that show the worst performance. As a result, the average rank of our model outperforms other models. We see that our model can generalize properties of molecules by not showing biased results on various specific tasks. We also compared our model with SMILESBERT. Our model gives better performance on most downstream tasks. Therefore, our matrix embedding layer and pre-training tasks effectively work on molecular properties prediction.

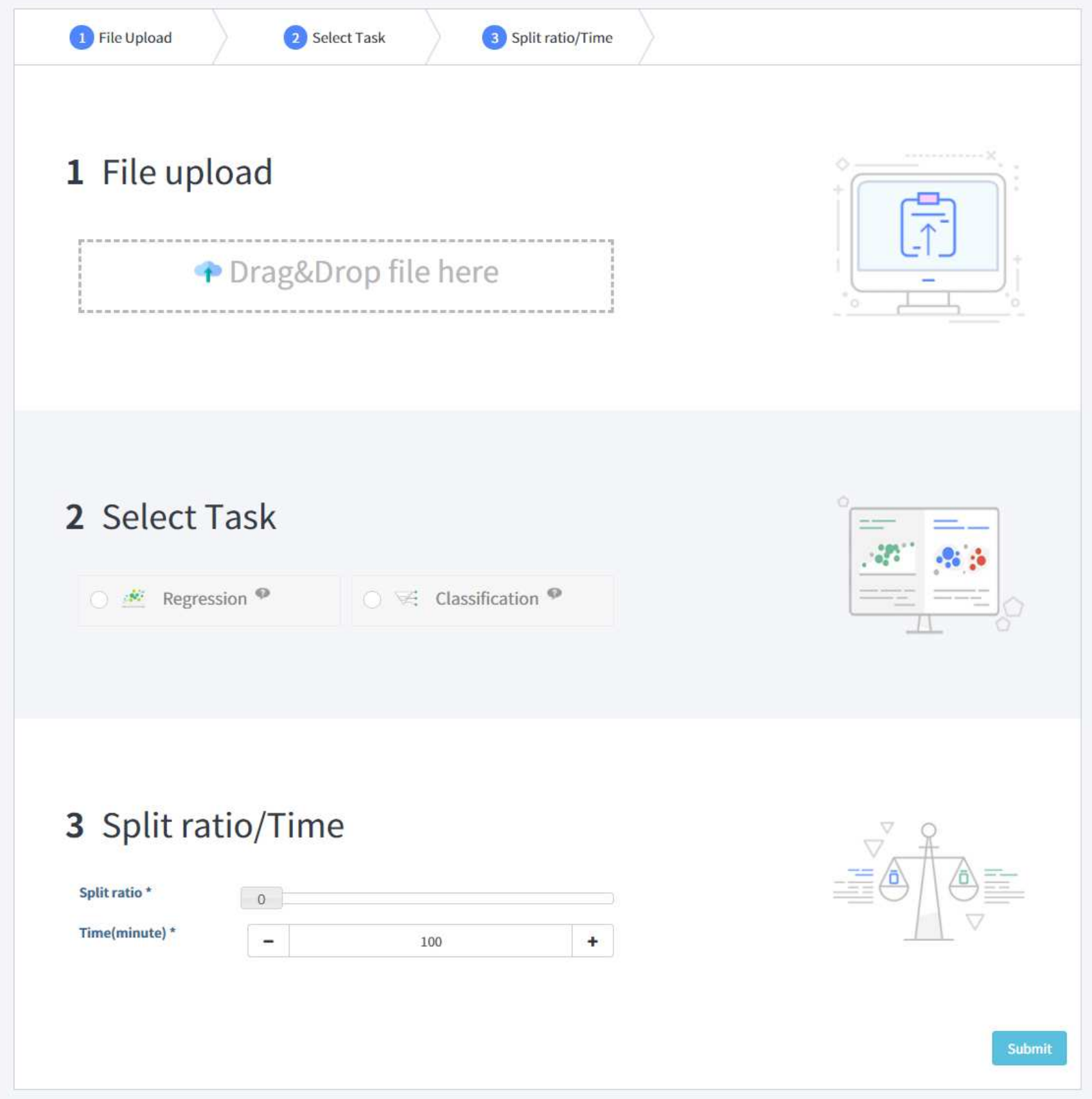

Figure 4. Job submission page of the fine-tuning service. Users upload their own dataset and select the type of task. There is no specific parameter selection for training.

We develop the fine-tuning service based on our pre-trained model shown as Figure 4. We design the service to utilize a characteristic of the pre-trained model that converges faster than a non-pretrained model. For using the service, users should prepare a dataset which contains SMILES and label of data in CSV format. Before job submission, users select the type of task, data split ratio, and time limitation. We minimize the optional parameters for fine-tuning to provide a user-friendly service. 


$\begin{array}{llll}\text { optimizer } & \text { batch size } & \text { learning.rate } & \text { metric } \\ \text { Adam } & 64 & 1.0 \mathrm{E}-5 & \text { RMSE } \\ \text { model } & \text { dropout } & \text { epoch } & \text { best. score } \\ \text { Transformer } & 0 & 15 & 1.30498 \\ & & & \text { Test Score }\end{array}$
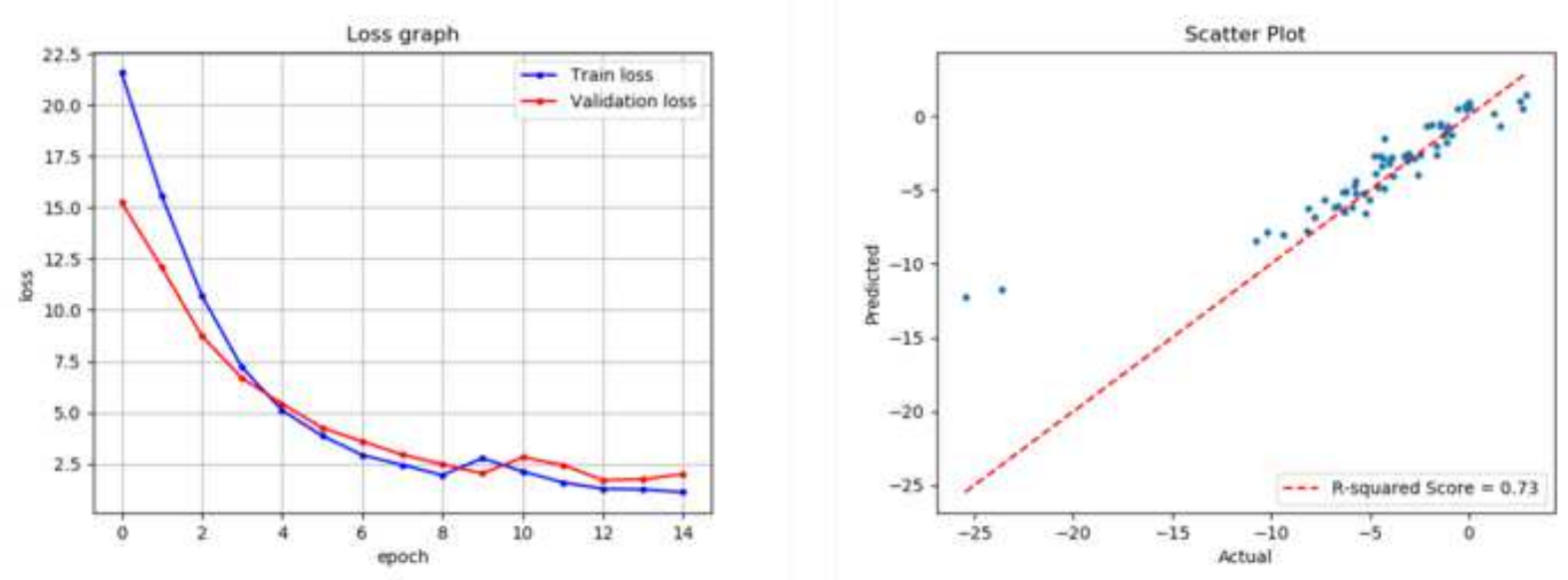

File Downioad

List View

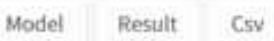

Figure 5. The result of the fine-tuning service. The result contains hyperparameters of the model, loss graph and performance on test set. Users can export the result and the model.

After job submission, users can get the result of training and performance on submitted data shown as Figure 5. We expect three contributions from the fine-tuning service. First, users do not have to spend lots of time and cost to pre-train the model. By using the service, users can skip the pre-training stage. The service also contains the pre-processing logic. Therefore, the user does not have to design additional processing logic. Second, users do not have to design a model specifically and they can train various molecular datasets. Using the service, they can build the model without domain knowledge on data and machine learning. Finally, users can obtain stable and high performance. As seen from our experiment results, we constructed a generalized model that can perform various molecular properties predictions. They can easily access the service by submitting datasets and get a reliable result. Users can start their new research or get insights about data by building their model via our service. We expect our service can lower the barrier of artificial intelligence for chemistry researchers.

\section{Conclusions}

Our work suggests constructing a robust pre-trained model by effectively integrating various representations from molecules. We proposed a novel self-supervised learning method merging various data representations for molecular properties prediction. Based on the Transformer, we designed the matrix embedding layer for learning connectivity and added a QED prediction task in pre-training. Our method achieved better average performance than other models on downstream tasks and showed stable performance on various data. From the result, it has been shown that our model learns the general properties of molecules well. We also implemented the fine-tuning service to provide a user-friendly training environment with a pre-trained model. We expect users could save their effort and costs for constructing and training the prediction model. Users can easily train the model without hyperparameter-tuning and get the result from our service. We make one step forward on designing a novel representation of molecules and utilizing the model in various fields via the fine-tuning service. For future work, we plan to device a representation that can import 3D structures or distance and apply our service to drug discovery processes such as virtual screening and drug-target interaction. 


\section{Data Availability}

The datasets used in pre-training and evaluation are available at ZINC and MoleculeNet. The source code of our work is available at https://github.com/HyunSeobKim/MergedBERT. The service will open to the public later.

\section{References}

1. Chen, H., Engkvist, O., Wang, Y., Olivecrona, M. \& Blaschke, T. The rise of deep learning in drug discovery. Drug discovery today 23, 1241-1250 (2018).

2. Gawehn, E., Hiss, J. A. \& Schneider, G. Deep learning in drug discovery. Mol. informatics 35, 3-14 (2016).

3. Zhang, L., Tan, J., Han, D. \& Zhu, H. From machine learning to deep learning: progress in machine intelligence for rational drug discovery. Drug discovery today 22, 1680-1685 (2017).

4. Lan, Z. et al. Albert: A lite bert for self-supervised learning of language representations. arXiv preprint arXiv:1909.11942 (2019).

5. Devlin, J., Chang, M.-W., Lee, K. \& Toutanova, K. Bert: Pre-training of deep bidirectional transformers for language understanding. arXiv preprint arXiv:1810.04805 (2018).

6. Yang, Z. et al. Xlnet: Generalized autoregressive pretraining for language understanding. In Advances in neural information processing systems, 5753-5763 (2019).

7. Vaswani, A. et al. Attention is all you need. In Advances in neural information processing systems, 5998-6008 (2017).

8. Hu, W. et al. Strategies for pre-training graph neural networks. arXiv preprint arXiv:1905.12265 (2019).

9. Glen, R. C. et al. Circular fingerprints: flexible molecular descriptors with applications from physical chemistry to adme. IDrugs 9, 199 (2006).

10. Rogers, D. \& Hahn, M. Extended-connectivity fingerprints. J. chemical information modeling 50, 742-754 (2010).

11. Hirohara, M., Saito, Y., Koda, Y., Sato, K. \& Sakakibara, Y. Convolutional neural network based on smiles representation of compounds for detecting chemical motif. BMC bioinformatics 19, 526 (2018).

12. Kimber, T. B., Engelke, S., Tetko, I. V., Bruno, E. \& Godin, G. Synergy effect between convolutional neural networks and the multiplicity of smiles for improvement of molecular prediction. arXiv preprint arXiv:1812.04439 (2018).

13. Duvenaud, D. K. et al. Convolutional networks on graphs for learning molecular fingerprints. In Advances in neural information processing systems, 2224-2232 (2015).

14. Li, J., Cai, D. \& He, X. Learning graph-level representation for drug discovery. arXiv preprint arXiv:1709.03741 (2017).

15. Xu, Z., Wang, S., Zhu, F. \& Huang, J. Seq2seq fingerprint: An unsupervised deep molecular embedding for drug discovery. In Proceedings of the 8th ACM international conference on bioinformatics, computational biology, and health informatics, 285-294 (2017).

16. Mikolov, T., Sutskever, I., Chen, K., Corrado, G. S. \& Dean, J. Distributed representations of words and phrases and their compositionality. In Advances in neural information processing systems, 3111-3119 (2013).

17. Quan, Z. et al. A system for learning atoms based on long short-term memory recurrent neural networks. In 2018 IEEE International Conference on Bioinformatics and Biomedicine (BIBM), 728-733 (IEEE, 2018).

18. Goh, G. B., Hodas, N. O., Siegel, C. \& Vishnu, A. Smiles2vec: An interpretable general-purpose deep neural network for predicting chemical properties. arXiv preprint arXiv:1712.02034 (2017).

19. Jaeger, S., Fulle, S. \& Turk, S. Mol2vec: unsupervised machine learning approach with chemical intuition. J. chemical information modeling $\mathbf{5 8}, 27-35$ (2018).

20. Honda, S., Shi, S. \& Ueda, H. R. Smiles transformer: Pre-trained molecular fingerprint for low data drug discovery. arXiv preprint arXiv:1911.04738 (2019).

21. Wang, S., Guo, Y., Wang, Y., Sun, H. \& Huang, J. Smiles-bert: large scale unsupervised pre-training for molecular property prediction. In Proceedings of the 10th ACM International Conference on Bioinformatics, Computational Biology and Health Informatics, 429-436 (2019).

22. Maziarka, Ł. et al. Molecule attention transformer. arXiv preprint arXiv:2002.08264 (2020).

23. Coley, C. W., Barzilay, R., Green, W. H., Jaakkola, T. S. \& Jensen, K. F. Convolutional embedding of attributed molecular graphs for physical property prediction. J. chemical information modeling 57, 1757-1772 (2017). 
24. Coley, C. W. et al. A graph-convolutional neural network model for the prediction of chemical reactivity. Chem. science 10, 370-377 (2019).

25. Bécigneul, G., Ganea, O.-E., Chen, B., Barzilay, R. \& Jaakkola, T. Optimal transport graph neural networks. arXiv preprint arXiv:2006.04804 (2020).

26. Wang, S. et al. Molecular property prediction based on a multichannel substructure graph. IEEE Access 8, 18601-18614 (2020).

27. Ma, H. et al. Dual message passing neural network for molecular property prediction. arXiv preprint arXiv:2005.13607 (2020).

28. Gilmer, J., Schoenholz, S. S., Riley, P. F., Vinyals, O. \& Dahl, G. E. Neural message passing for quantum chemistry. arXiv preprint arXiv:1704.01212 (2017).

29. Wu, Z. et al. Moleculenet: a benchmark for molecular machine learning. Chem. science 9, 513-530 (2018).

30. Bickerton, G. R., Paolini, G. V., Besnard, J., Muresan, S. \& Hopkins, A. L. Quantifying the chemical beauty of drugs. Nat. chemistry 4, 90-98 (2012).

31. Sterling, T. \& Irwin, J. J. Zinc 15-ligand discovery for everyone. J. chemical information modeling 55, 2324-2337 (2015).

32. Hendrycks, D. \& Gimpel, K. Gaussian error linear units (gelus). arXiv preprint arXiv:1606.08415 (2016).

33. Kingma, D. P. \& Ba, J. Adam: A method for stochastic optimization. arXiv preprint arXiv:1412.6980 (2014).

34. Martins, I. F., Teixeira, A. L., Pinheiro, L. \& Falcao, A. O. A bayesian approach to in silico blood-brain barrier penetration modeling. J. chemical information modeling 52, 1686-1697 (2012).

35. Challenge, T. D. Tox21 data challenge 2014 (2014).

36. Richard, A. M. et al. Toxcast chemical landscape: paving the road to 21 st century toxicology. Chem. research toxicology 29, 1225-1251 (2016).

37. Kuhn, M., Letunic, I., Jensen, L. J. \& Bork, P. The sider database of drugs and side effects. Nucleic acids research 44, D1075-D1079 (2016).

38. Novick, P. A., Ortiz, O. F., Poelman, J., Abdulhay, A. Y. \& Pande, V. S. Sweetlead: an in silico database of approved drugs, regulated chemicals, and herbal isolates for computer-aided drug discovery. PLoS One 8, e79568 (2013).

39. Gardiner, E. J., Holliday, J. D., O’Dowd, C. \& Willett, P. Effectiveness of 2 d fingerprints for scaffold hopping. Futur. medicinal chemistry 3, 405-414 (2011).

40. Subramanian, G., Ramsundar, B., Pande, V. \& Denny, R. A. Computational modeling of $\beta$-secretase 1 (bace-1) inhibitors using ligand based approaches. J. chemical information modeling 56, 1936-1949 (2016).

41. Delaney, J. S. Esol: estimating aqueous solubility directly from molecular structure. J. chemical information computer sciences 44, 1000-1005 (2004).

42. Mobley, D. L. \& Guthrie, J. P. Freesolv: a database of experimental and calculated hydration free energies, with input files. J. computer-aided molecular design 28, 711-720 (2014).

43. Liaw, A., Wiener, M. et al. Classification and regression by randomforest. R news 2, 18-22 (2002).

44. Xu, K., Hu, W., Leskovec, J. \& Jegelka, S. How powerful are graph neural networks? arXiv preprint arXiv:1810.00826 (2018).

\section{Acknowledgements}

This research was supported by the KISTI program (No. K-20-L02-C05-S01).

\section{Author contributions statement}

H.K. conceived the experiments. H.K. and J.L. conducted the experiments. S.A. and J.R.L supervised this work. All authors reviewed the manuscript.

\section{Additional information}

Competing Interests: The authors declare no competing interests 


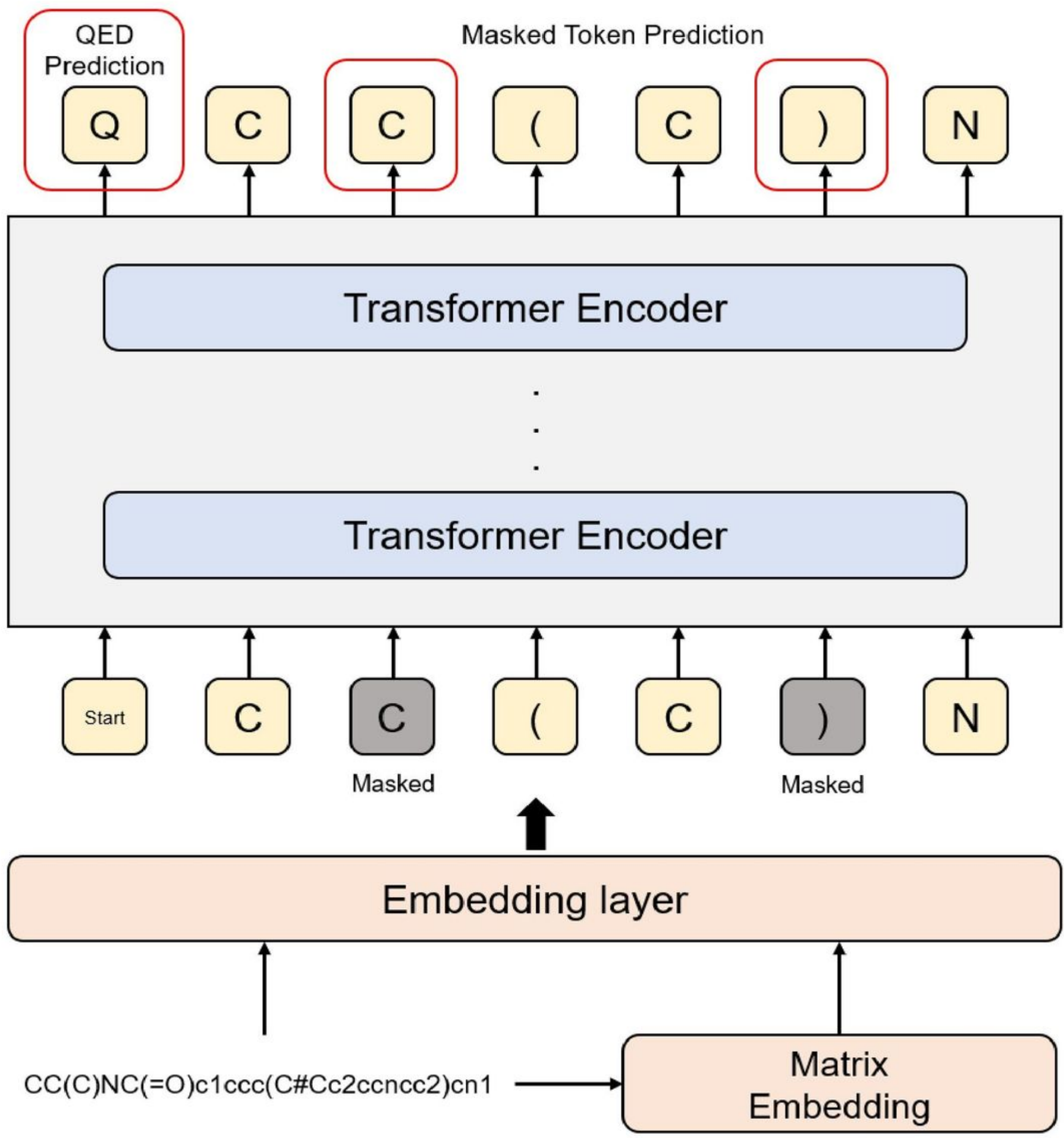

Figure 1

The overview of our pre-training methods and model. The input SMILES is converted to input vectors via embedding layer. The red boxes are targets of pre-training among the outputs of Transformer. The finetuning uses the same architectures except output layers. 
Input
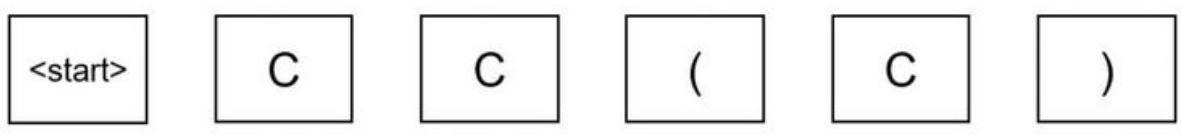

$\mathrm{N}$

\section{Token}

Embedding
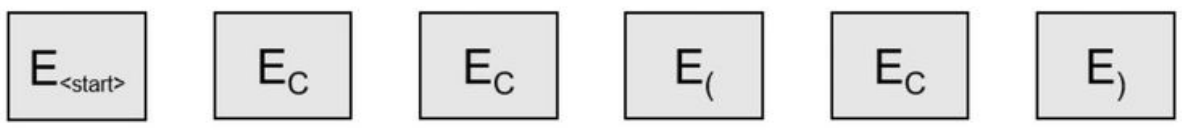

\section{$E_{N}$}

Position

Embedding
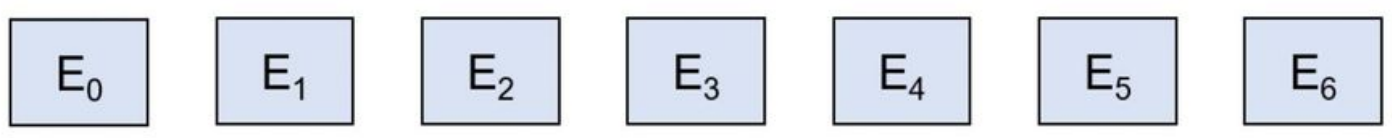

Matrix

Embedding
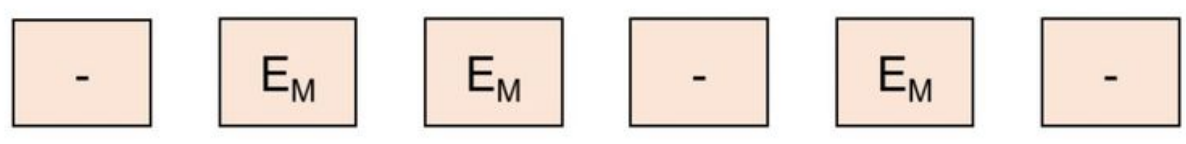

\section{$E_{M}$}

\section{Figure 2}

The input representation of SMILES. The input is the sum of the token embeddings, the position embeddings, and the matrix embeddings. The matrix embedding is only applied to atomic tokens.
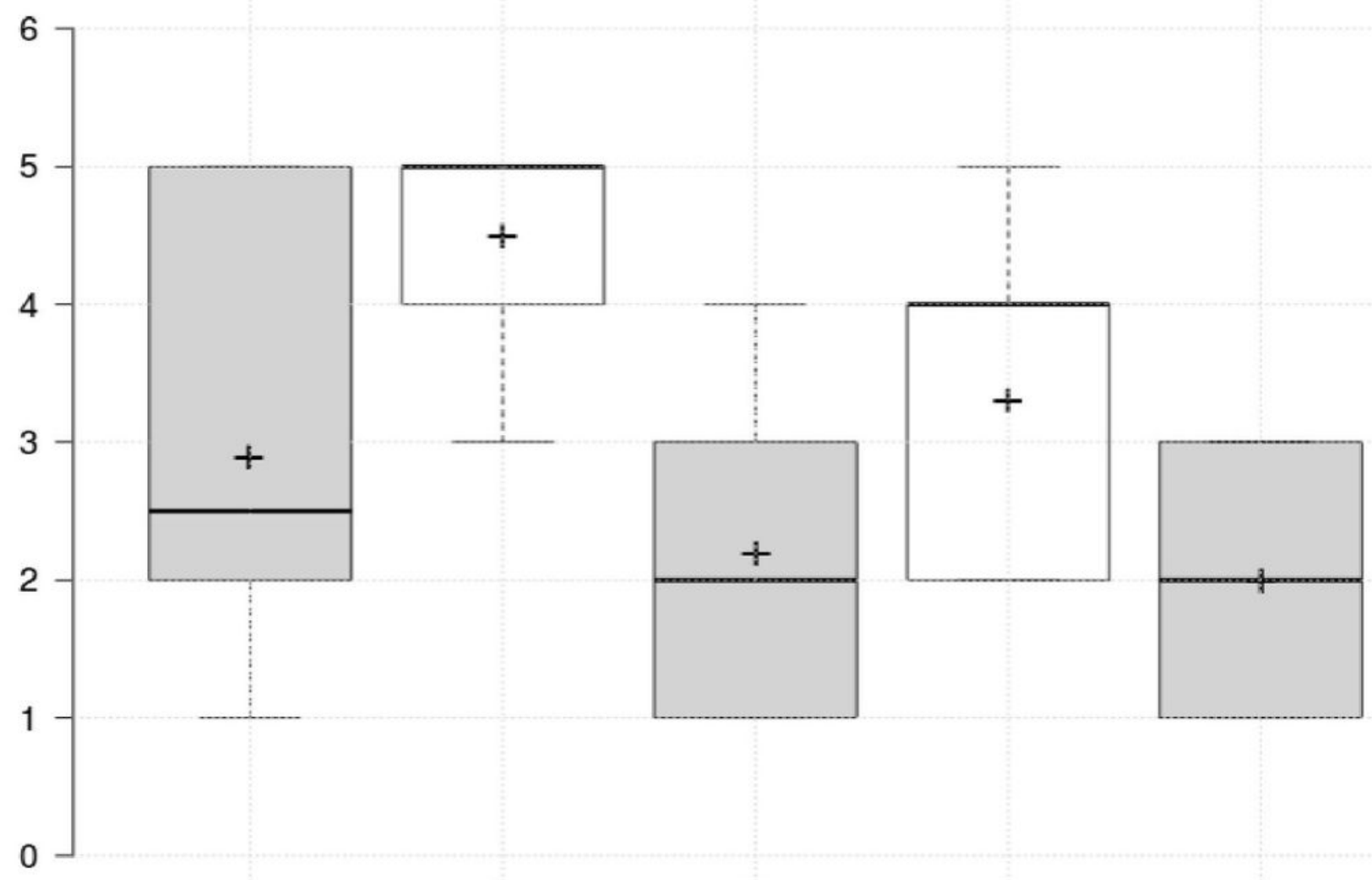

RandomForest

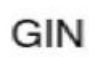

Pretrained-GIN SMILES-BERT

Our model

Figure 3 
The average rank across the 10 datasets from MoleculeNet. Our model outperforms other models. Interestingly, RandomForest records higher average rank than GIN and SMILES-BERT.

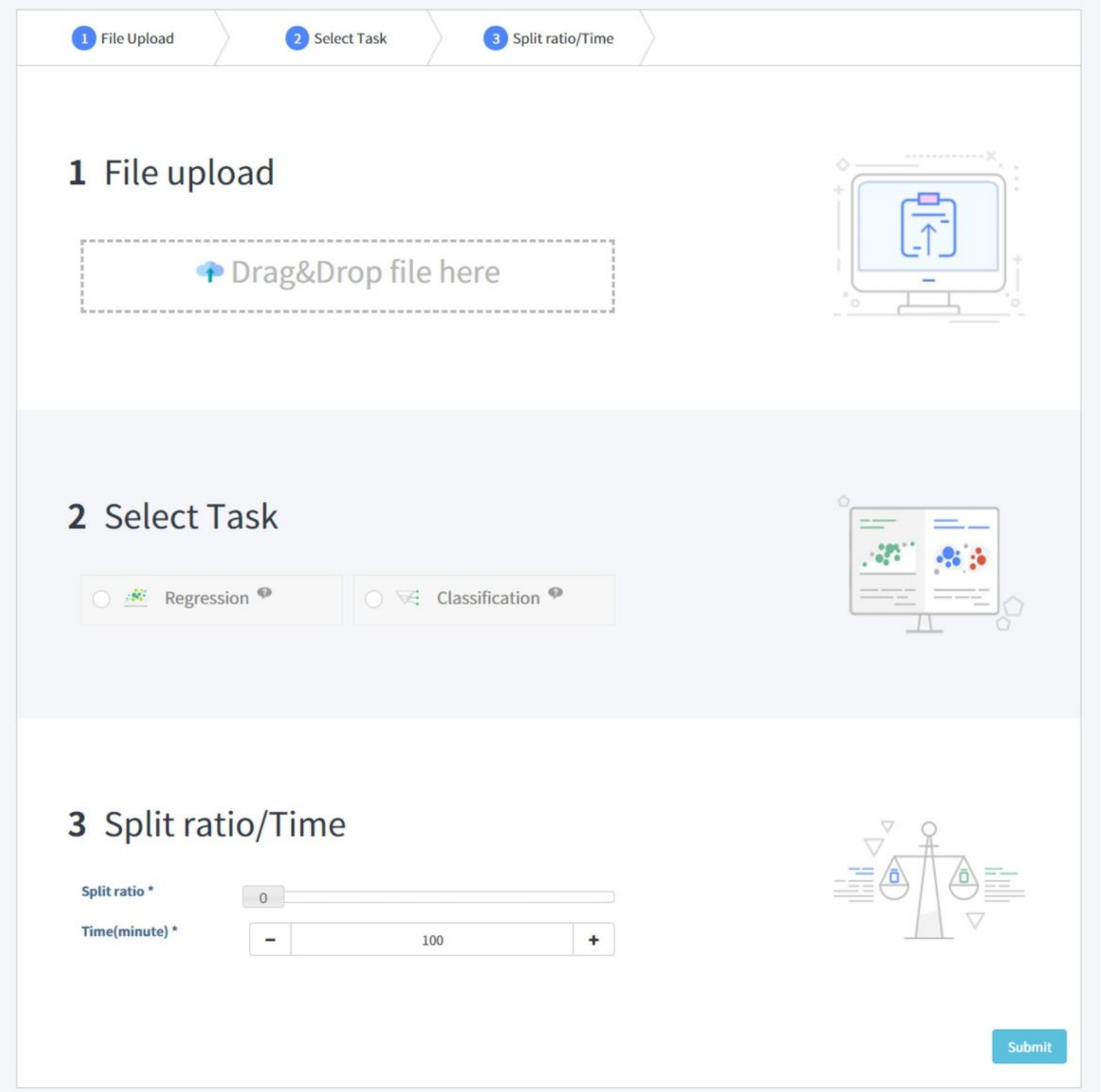

\section{Figure 4}

Job submission page of the fine-tuning service. Users upload their own dataset and select the type of task. There is no specific parameter selection for training. 


$\begin{array}{llll}\text { optimizer } & \text { batch_size } & \text { learning_rate } & \text { metric } \\ \text { Adam } & 64 & 1.0 E-5 & \text { RMSE } \\ \text { model } & \text { dropout } & \text { epoch } & \text { best_score } \\ \text { Transformer } & 0 & 15 & 1.30498\end{array}$

Loss Graph

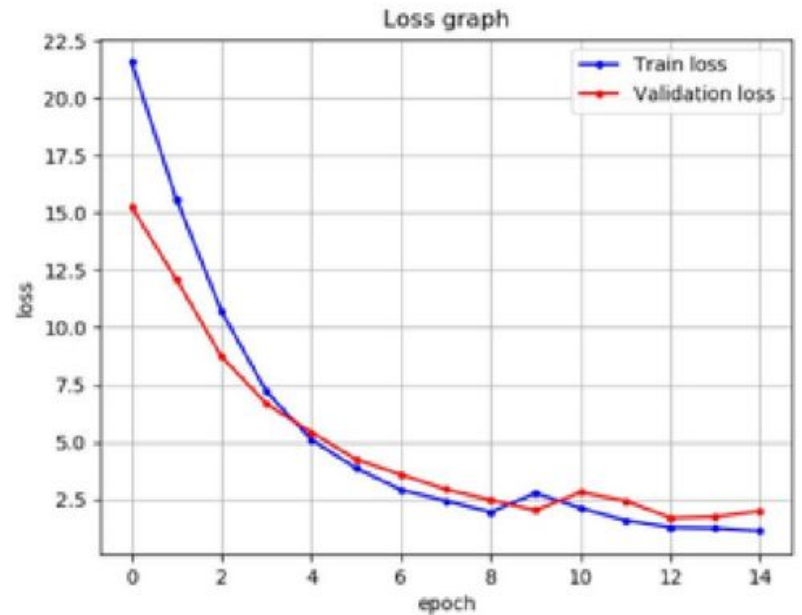

File Download

Model Result Cs:
Test Score

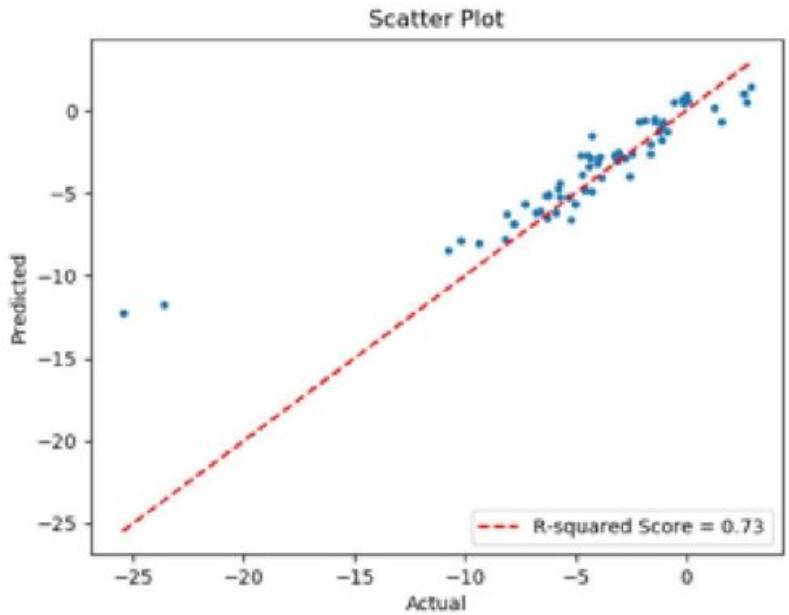

List View

国ist

\section{Figure 5}

The result of the fine-tuning service. The result contains hyperparameters of the model, loss graph and performance on test set. Users can export the result and the model. 